\title{
LA TOPONÍMIA \\ DE POLINYÀ DEL VALLÈS \\ ELS SEGLES X, XI I XII*
}

\author{
Jesús Alturo i Perucho
}

Polinyà és un municipi del Vallès Occidental limitat pels termes de Sentmenat i Palau-solità al nord, Santa Perpètua de Mogoda al sud $\mathrm{i}$ sud-est, Sabadell a l'oest i per la riera de Caldes a l'est ${ }^{1}$. L'antiguitat del poble és considerable, ja que cal buscar el seu origen en una antiga villa romana del Baix Imperi, tal com I'etimologia del topònim indica ${ }^{2}$. Amb tot, la seva història reconstruible per testimonis escrits no comença, pel que sabem, abans del 15 d'abril del 969.

- Agraeixo a la Sra. Isabel Izquierdo, antiga directora del Museu de Polinyà, al seu president actual, Sr. Llorenç Solà, i a la Sra. Rosa Estadella llur col-laboració en la tasca d'identificació dels topònims conservats encara avui.

1 Veg. J. PAYOLA, «Polinyà del Vallès», dins Gran geografia comarcal de Ca. talunya, vol. 6, Barcelona 1982, p. 187-190.

2 Aquesta és l'opinió de J. BALARI, Origenes históricos de Cataluña, vol. I, Abadía de San Cugat del Vallés $1964^{2}$, p. 39, que fa venir el nom de Paulinianum. I, en efecte, el primer cop que apareix Polinyà en la documentació és sota la forma de Pauleniano i precedit del terme villa. Aquesta denominació, que ja ens parla del seu origen, reapareix una altra vegada. En tots els altres textos, però, s' hi fa referència amb el nom de locus i més sovint amb el de terminus, però quan s'esmenta Sant Salvador de Polinyà hom parla sempre de parrachia. Hi ha uns quants casos, finalment, en què el nom de la població no ve precedit de cap altra especificació. 
És una escriptura d'aquesta data la que enregistra per primera vegada el nom del nostre poble arran de la venda que els comtes de Barcelona, Borrell II i Letgarda, feren a Galí dels drets públics damunt aquell ${ }^{3}$. Aquesta acta és certament interessant des de molts punts de vista, però ara volem ressaltar que hi van quedar ben detallats els límits del terme de Polinyà el segle X. Eren aquests: Arraona i Canyelles a la banda septentrional, Palau-solità i Figueroles a l'oriental, Omet i Santa Maria Antiga (Santiga) a la meridional i novament Arraona a l'occidental ${ }^{4}$.

Tot i que algun d'aquests topònims no ha perdurat fins als nostres dies ${ }^{5}$, és fàcil de veure-hi una coincidència amb les afrontacions actuals i, malgrat petites modificacions en els punts de referència, aquests termes es repeteixen en diplomes dels segles XI i XII. Vegem-ho: en una acta de venda de 1044, els termes de Polinyà queden circumscrits pels de Marata al nord, la riera de Caldes a l'est, la parròquia de Santa Maria Antiga i Omet al sud, i Arraona a 1'oest ${ }^{6}$; en un acord del 1055 , els límits respectius vénen determinats pels de la parròquia de Sentmenat i Canyelles, Palau-solità i la riera de Caldes, Santa Maria Antiga i Santa Perpètua de Mogoda,

Sobre I'etimologia de Polinyà, també en tractà P. AEBISCHER, «Etudes de toponymie catalanes, Memories de l'Institut d'Estudis Catalans, t. I, Barcelona 1926, p. 122.

${ }^{3}$ Sobre el passat historic de Polinyà, veg. el meu Diplomatari de Polinyà del Vallès. Aproximació a la història d'un poble del segle $X$ al XII, Bellaterra, $1985 \mathrm{i}$ «Polinyà del Vallès del segle $\mathrm{x}$ al XI. Estudi històric i diplomàtico-paleogràfic», Arrahona, II època, núm. 17 (1985), pp. 23-62.

4 Veg. el doc. 1 del meu Diplomatari cit., on el text llatí diu: «vindimus tibi villa que dicitur Pauleniano... Adfrontat de parte circi in termino de Arraona vel de Canellas et de oriente in terminio de Palacio Zalatan vel de Figariolas, de meridie in terminio de Olmedo vel de Sancta Maria, de occiduo similiter in terminio de Arraona.

Tots els documents citats en aquest article porten el número de la nostra edició. La xifra del costat correspon a l'any.

5 Sobre la seva localització i testimonis, veg. més endavant I'apartat dedicat als loci.

6 Veg. el doc. 17: «de parte orientis in rio Calles, de meriodie in termino de Sancta Maria Antiga siue in Olmedo, de occiduo in termine Araona, de circi in locum que dicunt Meserataw. 
i Sant Feliu d'Arraona7; en una permuta d'entorn de $1055^{8}$, hom assenyala com a línia divisòria la que passava per Arraona i Canyelles, Palau-solità i el camí públic que travessava Ullostet i el feu de Barberà, i la riera de Canyameres i Arraona9; en una nova venda de 1068 hom fixa els límits en Santa Maria de Palau-solità, la riera de Caldes, el camí que passant per Omet anava de Santa Maria Antiga a Granollers i el feu d'Arraona ${ }^{10}$; i, finalment, en una tercera venda d'una data pròxima a 119711, marquen els confins de Polinyà els corresponents de Sentmenat, la riera de Caldes i Santa Perpètua de Mogoda, Vall d'Omet i Santa Maria Antiga, i el castell d'Arraona'12.

Però la documentació referent a Polinyà del Vallès pervinguda fins avui no sols ens possibilita de dibuixar el cercle dels seus límits medievals, sinó que també ens serva el record de distints topònims ja perduts o dóna testimoniatge de la vellúria d'altres encara vius ${ }^{13}$.

7 Veg. el doc. 25: «de parte orientis terminant in Palacio Salatani et in rio de Calidas, a parte uero circii in terminum parrochia Sancti Minati et in Cannellas, de meridie in termine parrochie Sancte Marie Antique et Sancte Perpetue, de occiduo in termine parrochie Sancti Felicis Arraonaw.

${ }^{8}$ El pergamí està deteriorat a l'altura del cronograma, però, sens dubte, la data del document deu ésser pròxima a 1055. Per a aquest particular, veg. Diplomatari cit., p. 29.

9 Veg. el doc. 26: «de parte circii in terminio de Arraona seu de Chanellas, de aquilonis in Palacio Salatani siue in esirada pablica qui pengit per sumitate de ipsa serra de Figeroles, de meridie in estrada publica qui per-git per Ulmed siue in ipso feuuo de Barberano, de occiduo in rio de Channameres siue in termine de Arraonas.

$10 \mathrm{Veg}$. el doc. 47: ede oriente in alueo riul Callense, de meridie in stratam ubique pergente de Sancta Maria Antiqua usque ad Granullarias et transiir per Olmeti, de occiduo in feuum Arrahonensi, ex parte circi in termine (...) Sancte Marie Palacii Salatani».

11 El pergamí també està deteriorat a l'altura del cronograma, però la data és d'entorn de 1197 o, en tot cas, posterior a 1186. Per a aquest particular, veg. Diplomatari cit., p. 61.

12 Veg. el doc. 115: *ab oriente in riuo Calidarum et in parrochia de Sancta Perpetua, a meridie in Valle Olmeti et in parrochia de Antica, ab occiduo in termino castri de Arraonam, a cersio in termino castri Sancti Minati .

${ }^{13}$ La relació de topònims vindrà encapçalada sempre per la forma catalana actual. En el cas que el nom del lloc no hagi perdurat fins avui, dono també la forma presumiblement catalana a què correspondria la llatina, però precedida aleshores d'un asterisc. 
Destaquem, en primer lloc, que el nom de Polinyà, com és logic, és el més ben documentat de la sèrie i que, a causa de l'ortografia vacil-lant dels escrivans de l'època, apareix sota les formes següents: Pauleniano, Pauliliano, Pauliniano, Polegnano, Poliano, Poligna, Polignano, Polina, Polinano, Polinia, Polinián, Poliniano, Polinianum, Polinna, Polinnano, Polinnáno, Politano [sic], Pollennano, Pulianno, Puliano, Puliniani Valensi -única i curiosa mostra de l'actual denominació completa-i Puliniano.

Dins el nucli de la població, hi constatem uns pocs topònims:

—*El Castellar de Polinyà (iusta ipso Chastellar, doc. 22, 1054; iusta ipso Castellar de Polignano, doc. 30, 1058). Era situat prop del camí públic, dins el terreny ocupat per la sagrera de l'església de Sant Salvador de Polinyà.

—*'O'Om (ad ipso Olmi, doc. 33, 1058). La seva localització era davant la porta de la predita església.

— El Vall Antic o els Valls Antics (ad ipso Vallo Antico; infra ipsos Uallos anticos, doc. 35, 1060). Aquest lloc era entorn de l'església de Sant Salvador.

Entre la toponímia de l'interior de la població, potser ens serà lícit d'incloure-hi l'església de Sant Salvador, citada directament per primera vegada el $1054^{14}$; el cementiri, esmentat per primer cop el 1058 (in ipso cimiterio Sancti Saluatoris, doc. 30); i la sagrera, testimoniada el 1057 (in sagrera Sancti Saluatoris, doc. 29), per bé que els sagrers ja s'esmenten des de 102815 .

En la documentació estudiada per nosaltres també hom cita per primera vegada l'església de Sant Martí de Polinyà. La data del text correspon a l'any $1145^{16}$.

Al costat d'aquests topònims, n'hi ha d'altres que eren loci fora del nucli estricte de població i estaven escampats per diferents

14 En el doc. 22 hom fa referència a l'església de Sant Salvador amb els vocables ecclesia i domus, i en el doc. 33 amb el de basilica.

15 Per a tot el que fa referència a la sagrera $i$ als distints propietaris de sagrers, veg. Diplomatari cit., pp. 77-78.

16 Aquest document, però, no s'ha conservat en original, sinó en regest d'un estudiós anònim del segle XVIII. 
punts del terme de Polinyà:

_ ${ }^{*}$ L'Aiguarela (ad ipsa Aguarela, doc. 12, 1031).

_ *Figueroles (Figariolas, doc. 1, 969; Figeroles, doc. 15, 1041). Aquest paratge, situat al costat de Palau-solità, algunes vegades apareix en la documentació com a locus independent de Polinyà i citat com al seu límit oriental, però en d'altres ocasions hom s'hi refereix com a lloc del terme de Polinyà o de la seva parròquia ${ }^{17}$. Cal notar, també, que l'indret conegut pel nom de la Vall de Figueroles s'inclou també dins els termes de la parròquia de Santa Maria de Palau-solità (in parrochia Sancta Maria Palacio Salatani, in uocatiuo locho ipsa ualle de Figgerolas, doc. 45, 1068). D'altra banda, Figueroles donava nom també a una serra (estrada publica qui pergit per sumitate de ipsa serra de Figeroles siue in Palacio Salatini, doc. 26, 1055), a un torrent (in torrente de Figeroles, doc. 76, 1106) i a un mas (el manso de Pono [sic] de Figuerolas $^{18}$, doc. 106,1163$)$.

- Fontanet (Fontanedum, doc. 122, 1196; de Fontaneto, doc. 6, 994). Aquest $110 c$, situat al sud de Palau-solità, és citat, com l'anterior, com a indret de la parròquia de Sant Salvador i terme fronterer de la Boada i de Polinyà mateix. El topònim perdura com a nom d'un mas: can Fontanet.

_ * Granell (Granello, doc. 57, 1083). Paratge del terme de Polinyà.

- Marata (Meserata, doc. 17, 1044). Paratge de la parròquia de

17 Aquesta vacil-lació s'observa també en altres paratges com Omet, Fontanet i Marata. L'explicació possiblement rau en el fet que els termes de la parròquia de Sant Salvador devien ésser una mica més amples que els de Polinyà en tant que poble.

18 Quan, com aquí, dono una forma castellana o catalana antiga és perquè el text llatí original s' ha perdut i sols en queda el record per l'extracte del seu contingut realitzat pel cèlebre canonge J. MARTi, Resumen de instruments del archiu de Santa Anna de Barcelona fet per -, canonge regular de las Avellanas, obra manuscrita del segle XVII, o bé per quatre autors anònims de la mateixa època que compilaren un Resumen del fondo de pergaminos distribuidos por letras. Sobre tots aquests particulars, veg. Ja meva obra L'Arxiu Antic de Santa Anna de Barcelona del 942 al 1200. Aproximació histórico-lingüística, 3 vols., Barcelona 1985. 
Sant Salvador. En una ocasió fa de límit septentrional d'una propietat dels termes de Polinyà i en una altra és situat entre els termes de Sentmenat i de Sant Salvador de Polinyà, i també a l'oest de la serra de Figueroles. El topònim perviu com a nom d'un mas: can Marata ${ }^{19}$.

- Omet (de Olmedo, doc. 1, 969; Olmeti, doc. 40, 1066; Ulmed, doc. 69, 1093). Generalment, aquest paratge és inclòs en el terme de la parròquia de Sant Salvador, però apareix per primera vegada com a límit de Polinyà per la banda meridional, al costat de Santiga. La documentació, a més a més, fa referència als caps d'Omet (in capite Olmeto, doc. 64, 1086), a Vall d'Omet (in Valle Olmeti, doc. 115, 1197), al riu d'Omet (in riui quam nucupant de Olmeto, doc. 65, 1086; in rio de Ulmed, doc. 69, 1093), i a un mas del mateix nom (mansus qui uocatur de Ulmeto, doc. 111, 1179). El topònim es conserva en ca n'Omet de Dalt i de Baix, actualment enderrocats, i en el nom d'una riera ${ }^{20}$.

- Palau (Palacii, doc. 22, 1054). Citat com a paratge de la parròquia de Sant Salvador.

- *Puig-Oriol (Pugo Oriol, doc. 14, 1031; Puio Oriol, doc. 51, 1074). Paratge de la parròquia de Sant Salvador.

- El Pujol (ad ipsum Puiol, doc. 118, 1194). Paratge de la parròquia de Sant Salvador. A Palau, hi ha un mas que duu aquest nom.

Altres punts de la toponímia de Polinyà són esmentats com a llocs de les susdites partides. Així:

Pujol.

- ${ }^{*} \mathrm{La}$ Coma (de ipsa Coma, doc. 118, 1194). En els termes del

— *Franquesa (in Francheda, doc. 118, 1194). També en els termes del Pujol.

- *Vinya Rodona (de Uinea Rotunda, doc. 22, 1054). En els termes de Palau.

19 Veg. més endavant la relació dels masos.

20 Aquest mas, altrament dit de Gausbert, és qualificat també de locus pels textos ( $\ll$ in locho quem uochant mansum Iozberti», doc. 117, 1188). 
Finalment, cal tenir present també un grup de topònims referits a paratges de la rodalia, fora ja del terme - encara que no sempre així considerats ${ }^{21}$ - , i citats com a indrets fronterers. Ultra els ja esmentats en parlar de les afrontacions del terme polinyanenc, són:

- La Boada (de ipsa Buada, doc. 6, 994). Terme fronterer del de Polinyà i Fontanet, al sud de Palau-solità. A Palau existeix can Boada.

- Canyameres (in Cannameras, doc. 76, 1106). Límit occidental d'un alou de Marata. Al terme de Sabadell, hom hi troba can Canyameres.

Els diplomes de Polinyà del Vallès també ens parlen sovint de masos. Els que he recollit són aquests:

- *El mas que fou de Bernat Gelmir (mansum qui fuit de Bernard Gelmir, doc. 78, 1112). Fou llegat per Guillem Bonfill de Santa Coloma a la seva filla Sança22.

_ *El mas de Guillem Ramon de Savall (mansum de Gillelmo Ramundi de ipsa Valle, doc. 78, 1112). Fou llegat per Guillem Bonfill de Santa Coloma a la seva filla Elliardis.

_ *El mas de Ponç (mansum de Poncio, doc. 78, 1112). Fou llegat per Guillem Bonfill de Santa Coloma a la seva filla Guillema.

- *El mas de Joan Bernat (manso de Juan Bernat, doc. 106, 1163). Fou definit per Guillem de Santa Coloma a la col-legiata de Santa Eulàlia del Camp.

- ${ }^{*}$ El mas de Pono de Figueroles (manso de Pono [sic] de Figuerolas, doc. 106, 1163). Fou definit per Guillem de Santa Coloma a Santa Eulalia del Camp23.

_ *El mas de Sarabara (manso de Sarabara, doc. 106, 1163). Fou definit per Guillem de Santa Coloma a Santa Eulàlia del Camp. Potser correspon a can Gavarra (?).

21 Vegeu els loci Omet, Fontanet i Marata,

22 Els Santa Coloma detingueren el senyoratge i la jurisdicció damunt Polinyà fins a la darreria del segle XII. Per a l'arbre genealògic d'aquesta família des del segle X, veg. Diplomatari cit., pp. 70-73.

23 Per a la historia d'aquesta col-legiata, veg. L'Arxiu Antic de Santa Anna de Barcelona cit., vol. I, pp. 137-180. 
— ${ }^{*} \mathrm{El}$ mas de Soler (manso Soler, doc. 106, 1163). Fou definit per Guillem de Santa Coloma a Santa Eulàlia del Camp.

- El mas que el prevere Joan tenia per la canònica de Barcelona a Marata (mansum... in loco uocitato Meserata, doc. 107, 1163). Fou definit pel sacerdot a la catedral. Actualment existeix can Marata 24.

- ${ }^{*}$ El mas que Ramon de Camp i la seva esposa Pocululla tenien per Guilabert de Cruilles (mansum quem tenent uoce Guilaberti de Crudies, doc. 109, 1172).

- El mas dit d'Omet o també de Gausbert (mansum qui uocatur de Ulmeto, doc. 111, 1179; in locho quem uochant mansum Iozberti, doc. 117, 1188). Aquest mas fou tinença de Pere Gausbert. El seu fill, Guillem Gausbert, vengué els seus drets, salvats els dels senyors, a la seva tia Rossa. Finalment, Pere Berenguer de Marata, fill d'aquesta, va vendre els seus drets a la seva germana Guillema i al seu espòs, Bernat de Mas, Actualment perdura el nom de ca n'Omet de Dalt i de Baix ${ }^{25}$.

- El mas que Pere donà en concepte d'esponsalici a la seva muller Joana (mansum... in loco nominato ad Meseratam, doc. 113, 1185). Can Marata26.

- ${ }^{*}$ El mas de la Vall o de Polinyà (mansum de ipsa Ualle, doc. 119, 1194; mansum de Puliniano, doc. 123, 1196). Joan de Vall el tenia per Guillema de Salavert, la qual el llegà en testament als seus fills Ramon i Berenguer.

- El mas que Gaufrid Guilabert tenia a Fontanet (mansum... in loco qui dicitur Fontanedum, doc. 122, 1196). Fou donat en feu a Berenguer de Soler. Es refereix a can Fontanet.

Possiblement també cal considerar masos els següents27:

- ${ }^{*}$ El mas de Bonfill Dac (usque ad mansione de Bonefili Dacco, doc. 41, 1066).

24 Veg, més avall el mas que Pere donà per esponsalici a la seva muller.

25 Veg. més amunt la relació de paratges.

26 Veg. el mas suara citat del sacerdot Joan.

27 En els nostres textos de vegades mansio i mansum són sinònims. 
- ${ }^{*} \mathrm{El}$ mas de Miró Malanyec (prope mansione de Mironi Malaiego, doc. 41, 1066).

No deixen d'ésser presents en els nostres textos les fortaleses i els molins. Així, hi trobem:

- La fortalesa de la Rovira (ipsam fortedam de ipsa Ruuira, doc. 54, 1075). Antiga propietat de Berenguer Guillem, que el seu fill Ramon Guillem donà a Berenguer Bonfill perquè la tingués per batllia durant deu anys. Segurament es tracta de can Rovira.

_ *Els molins de Besanta (molendini de Besanta, doc. 109, 1172). Ramon de Camp i la seva muller Pocululla els compraren a Guilla. Potser correspon a Pedra Santa (?).

Alguns accidents geogràfics del terme de Polinyà també apareixen en la documentació:

- *El Puig de l'església (prope ipso puio de ipsa ecclesia, doc. 39,1065 ). 1121).

— El Puig de la Llebre o Llebrer (in Puio Leporino, doc. 87,

_ *Puig-Oriol. Citat sempre com a locus.

— * La serra de Figueroles (ipsa serra de Figerolas, doc. 58, 1084). A l'est de Marata.

— La serra de Polinyà (in serra de Polinnano, doc. 87, 1121). Hi passava el camí antic que unia Santa Perpètua amb Pedra Santa.

També hi són presents els rius, torrents i rieres:

- La riera de Caldes (in rio Calidarum, doc. $115, \pm 1179$; in rio de Calidas, doc. 25, 1055; in rio Callense, doc. 47, 1068; in rio Calles, doc. 17, 1044). Citada sovint com a afrontació oriental del terme de Polinyà.

- La riera de Canyameres (in rio Channameres, doc. 26, \pm 1055 ). Citada també com a límit del terme polinyanenc 28 .

_ *El riu que passava prop de la masia de Miró Malanyec fins a la de Bonfill Dac (in rio qui excurrit prope mansione de Mironi

28 La riera de Canaberes trobada en un regest de Josep Martí (veg. doc. 14) deu correspondre a la de Canyameres. 
Malaiego usque ad mansione de Bonefili Dacco uel ubique, doc. 41,1066$)$.

- El riu d'Omet (in rio de Ulmed, doc. 69, 1093). Citat com a afrontació oriental i occidental, respectivament, d'unes terres d'Omet.

- *La riera de Tirabous (viera de Tirabous, 1166). Prop de la riera de Caldes.

- ${ }^{*} \mathrm{El}$ torrent procedent de la Coma (in torrente qui excurrit de ipsa Coma, doc. 118, 1194). Citat com a afrontació septentrional d'un camp del Pujol.

- *El torrent de Figueroles (in torrente de Figeroles, doc. 76, 1106). Citat com a afrontació oriental d'un alou de Marata.

Cal deixar constàncía, també, que diverses vegades els textos esmenten rius o torrents innominats, que corresponen, sens dubte, als aquí referits.

Finalment, trobem testimoniades diferents vies de comunicació. Per sota de Polinyà passava una strata (strata que pergit subtus $P_{0}$ lliniano, doc. 67, 1089), que de vegades feia d'afrontació septentrional de propietats d'Omet (de circi in strada publica qui pergit subtus Sancti Saluatoris, doc. 40, 1066). Aquesta via arribava fins a l'església de Sant Salvador (uia -o strata en d'altres textosqui pergit a Sancti Saluatoris, doc. 11, 1030) i passava per la quintana de l'església (in ipsa estrada uel in ipsa chintana de Sancti Saluator, doc. 10,1028) i pel Castellar de Polinyà (iusta ipso Castellar de Polignano, prope strada publica, doc. 30, 1058).

També tenim notícia d'un camí que passava per Omet procedent de Santiga i en direcció a Granollers (strata ubique pergente de Santa Maria Antiqua usque ad Granullarios et transit per Olmeti, doc. 47,1068$)$. La mateixa via, límit oriental d'una propietat d'Omet, arribava a Santa Perpètua a través de la serra (strata qui pergit per ipsa serra ad Sancta Perpetua, doc. 69, 1093).

També Figueroles tenia vies d'accés, perquè l'afrontació occidental d'una finca del seu terme és un camí (ipsa strada, doc. 15,1041 , i strada publica, doc. 29,1057$)$. Aquesta via passava fins i tot per la serra de Figueroles (in strata ubique pergenti per ipsa serra de Figerolas, doc. 58, 1084). 
Per Granell, també hi passava un camí (in strata pergente per ipsa serra, doc. 57, 1083) j el mateix cas era per a Palau (in estrada pergenti ubique per ipsa serra, doc. 34,1059 ).

Remarquem, per acabar, que el topònim Polinyà era adjuntat també als noms dels seus fills quan aquests eren esmentats per algun personatge d'un altre poble ${ }^{29}$ o quan ells vivien fora del terme de la parròquia de Sant Salvador ${ }^{30}$, i també per desfer casos d'homonímia entre un veí del poble i un altre de fora ${ }^{31}$.

29 Aquest és el cas de Pere Adalbert de Polinyà (Petrus Adalberti de Puliianno, doc. 100, 1157), citat en el testament de Sança.

30 Aquest és el cas de Maiassenda de Polinyà (Maiasendis de Poliniano que uocant Malsignata, doc. 102, 1159), segurament habitant de la parròquia de Santa Maria de Caldes.

31 Aquest és el cas de Mir Bonfill de Polinyà (Mir Bonefili Polinianensi, doc. 66.1086 ) que es podia confondre amb Mir Bonfill (de Santa Coloma). 